\title{
Active multiple myeloma suppresses and typically eliminates coexisting MGUS
}

John P Campbell ${ }^{1,2,9}$, Jennifer L J Heaney ${ }^{1,9}$, Sankalp Pandya ${ }^{1}$, Zaheer Afzal ${ }^{1}$, Martin Kaiser ${ }^{3}$, Roger Owen ${ }^{4}$, J Anthony Child ${ }^{4}$, Walter Gregory ${ }^{5}$, Gareth J Morgan ${ }^{6}$, Graham H Jackson ${ }^{7}$, Chris M Bunce ${ }^{8}$ and Mark T Drayson ${ }^{*}, 1$

${ }^{1}$ Institute of Immunology and Immunotherapy, University of Birmingham, Birmingham B15 2TT, UK; ${ }^{2}$ Department for Health, University of Bath, Bath BA2 7AY, UK; Institute of Cancer Research, London SM2 5NG, UK; ${ }^{4}$ St James's University Hospital, Leeds LS9 7TF, UK; ${ }^{5}$ Clinical Trials Research Unit, University of Leeds, Leeds LS2 9NL, UK; ${ }^{6}$ The Myeloma Institute, University of Arkansas for Medical Sciences, Little Rock, AR 72205, USA; ${ }^{7}$ University of Newcastle, Newcastle upon Tyne NE1 7RU, UK and ${ }^{8}$ School of Biosciences, University of Birmingham, Birmingham B15 2TT, UK

Background: Myeloma is consistently preceded by premalignant monoclonal gammopathy of undetermined significance (MGUS). In > $5 \%$ of MGUS patients there is a second MGUS clone (biclonal gammopathy of undetermined significance; BGUS), yet, at myeloma diagnosis, presentation of biclonal gammopathy myeloma (BGMy) is considered less frequent, implying that myeloma eradicates coexisting MGUS.

Methods: In the largest study of its kind, we assessed BGMy frequency amongst 6399 newly diagnosed myeloma patients enrolled in recent UK clinical trials.

Results: Compared to expected prevalence (i.e., > 5\% of MGUS have BGUS), only 58 of 6399 (0.91\%) newly diagnosed myeloma patients had BGMy, indicating myeloma typically eliminates coexistent MGUS. In these 58 BGMy cases, the MGUS plasma cell clone was greatly suppressed in size compared to typical levels observed in conventional MGUS; contrarily, the MGUS clone did not inhibit the myeloma plasma cell clone in BGMy.

Conclusion: Myeloma eliminates the majority of competing MGUS, and when it does not, the MGUS clone is substantially reduced in size.

Myeloma and the asymptomatic precursor that consistently precedes it, monoclonal gammopathy of undetermined significance (MGUS) (Landgren et al, 2009; Weiss et al, 2009), are characterised by monoclonal plasma cells (PCs) in the bone marrow and monoclonal antibody (MAB) in blood. In a population-based study of 12482 persons, prevalence of MGUS was 3.7\% in blacks, $2.3 \%$ in whites and $1.8 \%$ in Hispanics, and within these MGUS populations, the prevalence of two MGUS clones-termed biclonal gammopathy of undetermined significance (BGUS) (Kyle et al, 1981)-was $15.4 \%, 6.8 \%$ and $12.8 \%$, respectively (Landgren et al, 2014). A proportion of new myeloma diagnoses also exhibit two MABs in immunofixation electrophoresis (IFE), termed myeloma with biclonal gammopathy (BGMy). This represents progression of one BGUS clone to active myeloma and the continued presence of a secondary MGUS clone (Kyle et al, 1981; Kyle et al, 2003). Given that the prevalence of MGUS in the general population-at the mean age of myeloma diagnosis-is $\sim 5 \%$ (Dispenzieri et al, 2010; Wadhera and Rajkumar, 2010), and prevalence of BGUS amongst all MGUS is $>5 \%$ (Landgren et al, 2014), one would also expect more than $5 \%$ of myeloma cases to be BGMy. The frequency of BGMy remains uncertain (Kyle et al, 1981; Nilsson et al, 1986; Riddell et al, 1986; Guastafierro et al, 2012; Garcia-Garcia et al, 2015). In a review of 1027 myeloma diagnoses in one centre, 2\% of cases were BGMy (Kyle et al, 2003). This lower than expected figure

\footnotetext{
*Correspondence: Professor MT Drayson; E-mail: M.T.Drayson@bham.ac.uk

${ }^{9}$ These authors contributed equally to this work.
}

Received 1 March 2017; revised 19 June 2017; accepted 22 June 2017; published online 20 July 2017

(C) 2017 Cancer Research UK. All rights reserved 0007-0920/17 
warrants validation as it implies that when myeloma arises in an individual with BGUS, the malignant myeloma clone eliminates the competing MGUS clone. Here, in the largest study of its kind, we assessed 6399 newly diagnosed myeloma patients entered into three multicentre UK clinical trials to determine the frequency, and $\mathrm{MAB}$ sizes of, BGMy at myeloma diagnosis.

\section{MATERIALS AND METHODS}

Trial patients. The BGMy patients were identified from 6399 newly diagnosed myeloma patients enrolled in one of the following multicentre, phase III trials: the UK MRC Myeloma IX trial ( $N=1693$; ISRCTN68454111); the CRUK Myeloma XI trial up to an induction randomisation date of 23 July $2015(N=3880$; ISRCTN49407852); or the UK NIHR TEAMM Trial up to a randomisation date of 23 July 2015 ( $N=826$; ISRCTN51731976). All patients gave written informed consent. The study was approved by the institutional review boards of the participating centres and was conducted according to the Declaration of Helsinki and the International Conference on Harmonization Guidelines for Good Clinical Practice.

Laboratory tests. The MABs in serum were identified by IFE (Sebia, Paris, France) and quantified by protein zone electrophoresis and densitometry (SPE; Interlab, Rome, Italy); please refer to Supplementary Information for more information. In patients with a light-chain (LC) MAB without a heavy-chain (HC) component (i.e., LC-only myeloma), MAB size was measured and monitored by involved free LCs (Binding Site, Birmingham, UK) expressed in $\mathrm{gl}^{-1}$.
Statistical analyses. Differences between groups were analysed by one-way ANOVA for continuous variables (e.g., MAB size), and $\chi^{2}$ for categorical variables and frequencies. One-sample $t$-tests were used to compare observed MAB sizes to previously observed mean MAB sizes in the literature; observed BGMy MAB sizes were compared to reported MAB sizes in MGUS (Turesson et al, 2014) and myeloma using a combined data set from MIX and MXI UK trials. Correlational analyses were conducted using Pearson's for normally distributed data and Spearman's Rank where data was skewed. Significance was accepted at $P<0.05$. Data were analysed by IBM SPSS statistics version 21 (Armonk, NY, USA).

\section{RESULTS}

Frequency of BGMy and demographics of BGMy patients. A total of 58 patients had BGMy (from $\mathrm{MIX}=18, \mathrm{MXI}=33$ and TEAMM =7) and 6341 had monoclonal gammopathy myeloma (MGMy) giving a BGMy frequency of 58 out of 6399 patients (0.91\%; $99 \%$ confidence interval: $\pm 0.3 \%$ ). Comparison of the 58 BGMy patients with MGMy patients in the same trials found no differences in age: BGMy median age (range) was 69 (45-86) years; and MGMy was 66 (28-90) years.

Distinguishing MGUS from myeloma MABs. The frequencies and concentrations of serum MABs in all 58 BGMy patients at trial entry are presented in Table 1; the largest MAB is classified as the myeloma $\mathrm{MAB}$ and is referred to as ' $\mathrm{M1}$ ', whereas the smaller MAB was classified as the MGUS MAB and is referred to as ' $M 2$ '. In 4 patients with a FLC $\mathrm{MAB}>500 \mathrm{mgl}^{-1}$ and intact

Table 1. Characteristics and frequencies of M1 and M2 in 58 BGMy patients at myeloma diagnosis, compared to expected frequencies and characteristics of MGMy and conventional MGUS

\begin{tabular}{|c|c|c|c|c|c|c|c|c|c|c|c|c|c|c|}
\hline \multicolumn{7}{|c|}{ MAB 1} & \multicolumn{8}{|c|}{ MAB 2} \\
\hline \multicolumn{2}{|c|}{$\mathrm{HC}$ isotype } & \multirow{4}{*}{$\begin{array}{c}\text { Frequency } \\
\text { (N) } \\
\\
39\end{array}$} & \multirow{4}{*}{$\begin{array}{c}\begin{array}{c}\text { Proportion } \\
(\%)\end{array} \\
\\
\\
\\
\\
\end{array}$} & \multirow{4}{*}{$\begin{array}{c}\text { Conc. }\left(\mathrm{gl}^{-1}\right) \\
\text { (median (range)) } \\
\\
{ }^{3} 36.1(4.8-70.0)\end{array}$} & \multicolumn{2}{|c|}{ MGMy reference ranges ${ }^{\mathrm{a}}$} & \multicolumn{2}{|c|}{$\mathrm{HC}$ isotype } & \multirow{4}{*}{$\begin{array}{c}\text { Frequency } \\
\text { (N) } \\
\\
\\
33\end{array}$} & \multirow{4}{*}{$\begin{array}{c}\begin{array}{c}\text { Proportion } \\
\text { (\%) }\end{array} \\
\\
\\
\\
\\
\\
\end{array}$} & \multirow{4}{*}{$\begin{array}{c}\text { Conc. }\left(\mathrm{gl}^{-1}\right) \\
\text { (median (range)) } \\
\\
\mathrm{d}_{2.6(0.5-12.0)}\end{array}$} & \multicolumn{3}{|c|}{ MGUS reference ranges ${ }^{b}$} \\
\hline \multirow[b]{3}{*}{$\lg G$} & \multirow[b]{3}{*}{ Total } & & & & \multirow{3}{*}{$\begin{array}{l}\text { Proportion } \\
\text { (\%) } \\
\\
\\
59.9\end{array}$} & \multirow{3}{*}{$\begin{array}{c}\text { Conc. }\left(\mathrm{gl}^{-1}\right) \\
\text { (median (range)) } \\
34.4(0.8-100.2)\end{array}$} & \multirow[b]{3}{*}{$\lg G$} & \multirow[b]{3}{*}{ Total } & & & & $\begin{array}{c}\text { Kyle et al, } \\
2006\end{array}$ & \multicolumn{2}{|c|}{ Turesson et al, 2014} \\
\hline & & & & & & & & & & & & \multicolumn{2}{|c|}{ Proportion (\%) } & $\begin{array}{l}\text { Conc. }\left(g \mathrm{l}^{-1}\right) \\
\text { (median } \pm \text { s.d.) }\end{array}$ \\
\hline & & & & & & & & & & & & 68.9 & 68.8 & $7.0 \pm 6.0$ \\
\hline & $\lg G_{\kappa}$ & 26 & 66.7 & $32.6(4.8-70.0)$ & & & & $\lg G \kappa$ & 17 & 51.5 & $2.5(0.5-12.0)$ & & & \\
\hline & $\lg G \lambda$ & 13 & 33.3 & $39.7(10.2-60.0)$ & & & & $\lg \mathrm{G} \lambda$ & 16 & 48.5 & $2.8(0.5-9.0)$ & & & \\
\hline \multirow[t]{3}{*}{$\lg \mathrm{A}$} & Total & 14 & 24.1 & ${ }^{c} 25.4(1.8-60.0)$ & 23.6 & $33.0(0.2-96.6)$ & $\lg \mathrm{A}$ & Total & 11 & 19.0 & $d_{1.7}(0.5-11.6)$ & 10.8 & 14.7 & $8.0 \pm 5.0$ \\
\hline & $\lg A \kappa$ & 8 & 57.1 & $26.9(1.8-60.0)$ & & & & $\lg A \kappa$ & 5 & 45.5 & $1.3(0.5-3.9)$ & & & \\
\hline & $\lg A \lambda$ & 6 & 42.9 & $23.2(3.9-53.0)$ & & & & $\lg A \lambda$ & 6 & 54.5 & $2.5(0.5-11.6)$ & & & \\
\hline \multirow[t]{3}{*}{$\lg D$} & Total & 1 & 1.7 & $2.6(2.6-2.6)$ & 1.7 & $4.2(0.2-36.9)$ & $\lg M$ & Total & 14 & 24.1 & $d_{1.3}(0.5-6.0)$ & 17.2 & 16.2 & $7.0 \pm 6.0$ \\
\hline & $\lg D_{k}$ & 0 & 0.0 & - & & & & $\lg M \kappa$ & 12 & 85.7 & $1.3(0.5-6.0)$ & & & \\
\hline & $\lg \mathrm{D} \lambda$ & 1 & 100 & $2.6(2.6-2.6)$ & & & & $\lg M \lambda$ & 2 & 14.3 & $1.6(0.6-2.7)$ & & & \\
\hline \multirow[t]{3}{*}{ FLC } & Total & 4 & 6.9 & $1.0(0.5-1.9)$ & 13.1 & $2.6(0.02-46.7)$ & & & & & & & & \\
\hline & $\mathrm{FLC} \kappa$ & 3 & 75.0 & $1.4(0.5-1.9)$ & & & & & & & & & & \\
\hline & $\mathrm{FLC} \lambda$ & 1 & 25.0 & $0.7(0.7-0.7)$ & & & & & & & & & & \\
\hline \multicolumn{2}{|c|}{ LC isotype } & Frequency $(N)$ & Proportion (\%) & $\begin{array}{c}\text { Conc. }\left(\mathrm{gl}^{-1}\right) \\
\text { (median (range)) }\end{array}$ & \multicolumn{2}{|c|}{ MGMy reference proportion ${ }^{\mathbf{a}}(\%)$} & \multicolumn{2}{|c|}{ LC isotype } & Frequency $(N)$ & Proportion (\%) & $\begin{array}{c}\text { Conc. }\left(\mathrm{gl}^{-1}\right) \\
\text { (median (range)) }\end{array}$ & \multicolumn{3}{|c|}{$\begin{array}{l}\text { MGUS reference proportion (\%) Kyle } \\
\text { et al, } 2006\end{array}$} \\
\hline \multicolumn{2}{|l|}{$\kappa$} & 37 & 63.8 & $26.0(0.5-70.0)$ & & 66.0 & & $\kappa$ & 34 & 58.6 & $1.8(0.4-12.0)$ & \multicolumn{3}{|c|}{62.0} \\
\hline \multicolumn{2}{|l|}{$\lambda$} & 21 & 36.2 & $27.0(0.7-60.0)$ & & 34.0 & & $\lambda$ & 24 & 41.4 & $2.7(0.5-11.6)$ & & 37.9 & \\
\hline \multicolumn{15}{|c|}{$\begin{array}{l}\text { Abbreviations: BGMy = biclonal gammopathy myeloma; Conc. = concentration; FLC = free } \\
\text { gammopathy myeloma; MGUS = monoclonal gammopathy of undetermined significance. } \\
{ }^{a} \text { Reference ranges aggregated from } 3248 \text { patients with MGMy diagnosed at entry into My } \\
{ }^{b} \text { Reference ranges derived from } 728 \text { MGUS patients (Turesson et al, 2014) or } 694 \text { MGUS } p \\
{ }^{c} \text { Significant difference between M1 and M2 concentration (IgG } P<0.001 \text {; IgA } P<0.001 \text { ). } \\
{ }^{d} \text { Significant difference between M2 concentration and expected MAB concentration in M }\end{array}$} \\
\hline
\end{tabular}


Table 2. Frequency of M1 and M2 combinations observed in BGMy

\begin{tabular}{|c|c|c|c|c|c|}
\hline \multirow[t]{2}{*}{ MAB 1} & \multirow[t]{2}{*}{ MAB 2} & \multicolumn{2}{|c|}{ MAB 2 frequency } & \multicolumn{2}{|c|}{ MAB 2 proportion } \\
\hline & & $\begin{array}{l}\text { Observed } \\
\text { BGMy } \\
\text { frequency } \\
(N)\end{array}$ & $\begin{array}{l}\text { Expected } \\
\text { MGUS }^{a} \\
\text { frequency } \\
(N)\end{array}$ & $\begin{array}{c}\text { Observed } \\
\text { BGMy } \\
\text { proportion } \\
(\%)\end{array}$ & $\begin{array}{c}\text { Expected } \\
\text { MGUS }^{\mathrm{a}} \\
\text { proportion } \\
(\%)\end{array}$ \\
\hline \multirow{6}{*}{$\begin{array}{l}\lg G \kappa \\
(N=26)\end{array}$} & $\lg G \kappa$ & $0^{b}$ & 12 & 0 & 46 \\
\hline & $\lg G \lambda$ & 10 & 6 & 38 & 23 \\
\hline & $\lg \mathrm{A} \kappa$ & 3 & 3 & 12 & 10 \\
\hline & $\lg A \lambda$ & 4 & 1 & 15 & 5 \\
\hline & $\lg M \kappa$ & 7 & 3 & 27 & 11 \\
\hline & $\lg M \lambda$ & 2 & 1 & 8 & 5 \\
\hline \multirow{6}{*}{$\begin{array}{l}\lg G \lambda \\
(N=13)\end{array}$} & $\lg G \kappa$ & 6 & 6 & 46 & 46 \\
\hline & $\lg \mathrm{g} \lambda$ & 0 & 3 & 0 & 23 \\
\hline & $\lg A \kappa$ & 2 & 1 & 15 & 10 \\
\hline & $\lg A \lambda$ & 2 & 1 & 15 & 5 \\
\hline & $\lg \mathrm{M} \kappa$ & 3 & 1 & 23 & 11 \\
\hline & $\lg M \lambda$ & 0 & 1 & 0 & 5 \\
\hline \multirow{6}{*}{$\begin{array}{l}\lg A \kappa \\
(N=8)\end{array}$} & $\lg G \kappa$ & 5 & 4 & 63 & 46 \\
\hline & $\lg G \lambda$ & 1 & 2 & 13 & 23 \\
\hline & $\lg A \kappa$ & 0 & 1 & 0 & 10 \\
\hline & $\lg A \lambda$ & 0 & 0 & 0 & 5 \\
\hline & $\lg M \kappa$ & 2 & 1 & 25 & 11 \\
\hline & $\lg \mathrm{M} \lambda$ & 0 & 0 & 0 & 5 \\
\hline \multirow{6}{*}{$\begin{array}{l}\lg A \lambda \\
(N=6)\end{array}$} & $\lg G \kappa$ & 4 & 3 & 67 & 46 \\
\hline & $\lg G \lambda$ & 2 & 1 & 33 & 23 \\
\hline & $\lg A \kappa$ & 0 & 1 & 0 & 10 \\
\hline & $\lg A \lambda$ & 0 & 0 & 0 & 5 \\
\hline & $\lg M \kappa$ & 0 & 1 & 0 & 11 \\
\hline & $\lg M \lambda$ & 0 & 0 & 0 & 5 \\
\hline
\end{tabular}

Abbreviations: $\mathrm{BGMy}=$ biclonal gammopathy myeloma; $\mathrm{FLC}=$ free light chain; $\mathrm{MAB}=$ monoclonal antibody; MGUS = monoclonal gammopathy of undetermined significance. Because of low frequency, data not shown from one patient with IgD MAB 1 and four patients with FLC MAB 1.

a Reference range proportions obtained from Turesson et al (2014), with assumed 2:1 proportion of kappa: lambda for each heavy-chain isotype.

${ }^{\mathrm{b}} \chi^{2}$-analyses revealed significant differences between the observed frequency of $\lg \mathrm{G} \kappa$ pairs in BGMy compared to those expected in MGUS $\left(P<0.001 ; \chi^{2}=15.60\right)$. A trend $(P=0.065$ : $\left.\chi^{2}=3.391\right)$ was observed for frequency of observed $\lg G \lambda$ pairs in BGMy and those expected in MGUS.

immunoglobulin $<5 \mathrm{gl}^{-1}$, the FLC MAB was selected as M1. M1s were 10 - to 20 -fold larger than $\mathrm{M} 2 \mathrm{~s}$ with median M1 IgG $34.4 \mathrm{gl}^{-1}$ compared to M2 IgG $2.6 \mathrm{gl}^{-1}$; and M1 IgA $33 \mathrm{gl}^{-1}$ compared to M2 IgA $1.7 \mathrm{gl}^{-1}$.

M1 and M2 MAB combinations in BGMy are presented in Table 2 where it can be seen that 37 out of 58 BGMy (65\%) combinations exhibited different LC isotypes; assessment of available serum FLC levels in these patients (illustrated in Supplementary Figure 1) demonstrated that elevated FLC levels were predominantly associated with the M1 clone.

In Table 2, we show that two independent IgG MABs was the most common M1 and M2 combination (16 out of 58 patients), but not as common as expected (27 out of 58), and we did not observe any patients with two IgG MABs with the same LC isotype ( 15 out of 58 expected; $P<0.001 ; \chi^{2}=17.228$ ). In 6 BGMy patients with two IgG MABs, the electrophoretic mobilities of the MABs
Table 3. Comparison of M1 and M2 concentrations in BGMy with expected MAB sizes in conventional MGMy and in MGUS

\begin{tabular}{|c|c|c|c|c|}
\hline Size & $\begin{array}{c}\text { M1 } \\
\text { proportion } \\
\text { and } \\
\text { frequency }^{\mathrm{a}}\end{array}$ & $\begin{array}{c}\text { Expected } \\
\text { proportion } \\
\text { in MGMy } \\
(\%)^{b, c}\end{array}$ & $\begin{array}{c}\text { M2 } \\
\text { proportion } \\
\text { and } \\
\text { frequency }\end{array}$ & $\begin{array}{c}\text { Expected } \\
\text { proportion } \\
\text { in MGUS } \\
(\%)^{\mathrm{d}}\end{array}$ \\
\hline$<4.99 \mathrm{gl}^{-1}$ & $7 \%(N=4)$ & 7 & $83 \%(N=48)$ & 24 \\
\hline $5.00-9.99 \mathrm{gl}^{-1}$ & $7 \%(N=4)$ & 5 & $14 \%(N=8)$ & 19 \\
\hline $10.00-14.99 \mathrm{gl}^{-1}$ & $7 \%(N=4)$ & 5 & $3 \%(N=2)$ & 33 \\
\hline $15.00-19.99 \mathrm{gl}^{-1}$ & $7 \%(N=4)$ & 6 & $0 \%(N=0)$ & 18 \\
\hline $20.00-24.99 \mathrm{gl}^{-1}$ & $13 \%(N=7)$ & 9 & $0 \%(N=0)$ & 5 \\
\hline$>25.00 \mathrm{gl}^{-1}$ & $57 \%(N=31)$ & 68 & $0 \%(N=0)$ & 1 \\
\hline \multicolumn{5}{|c|}{$\begin{array}{l}\text { Abbreviations: BGMy= biclonal gammopathy myeloma; MAB = monoclonal antibody; } \\
\text { MGMy= monoclonal gammopathy myeloma; MGUS=monoclonal gammopathy of unde- } \\
\text { termined significance. } \\
\text { Excludes four patients diagnosed with a FLC myeloma M1 (all four patients had a whole } \\
\text { MAB }<4.99 \mathrm{~g}^{-1} \text { ). } \\
\text { beference range represents aggregated data from patients with MGMy diagnosed at entry } \\
\text { into Myeloma IX and Myeloma XI trials. }\end{array}$} \\
\hline
\end{tabular}

were very similar and so these MABs were only reliably distinguishable by their different LC isotype. Accordingly, we may have missed a third of the 15 out of 58 expected IgG MAB pairs of the same LC isotype, because of electrophoretic mobility similarities.

MGUS MAB isotypes and levels in BGMy compared to conventional MGUS. The most frequent M2 was IgG (33 out of 58 ), and a quarter of M2 clones (14 out of 58) secreted IgM. The frequency of different M2 HC isotypes was closest to the patterns observed in two MGUS studies of 728 and 694 MGUS patients, rather than the different $\mathrm{HC}$ frequencies seen in myeloma (Kyle et al, 2006; Turesson et al, 2014) (Table 1). There were, however, less IgG M2s than would be expected in a typical MGUS population (33 versus 41) and more $\operatorname{IgM}$ and $\operatorname{IgA}$ isotypes (25 versus 16; trend observed: $P=0.092 ; \chi^{2}=2.83$ ).

The level of the MGUS-associated MAB (M2) in BGMy was smaller than sizes reported in MGUS (Turesson et al, 2014) by 2.5fold for IgG and 5-fold for IgA and IgM MABs $(P<0.001)$. Thus, whereas coexistent MGUS (i.e., M2) did not appear to suppress the myeloma clone (i.e., M1) in BGMy, the presence of myeloma (M1) significantly suppressed MGUS (M2) MAB levels. The magnitude of this suppression is illustrated in Table 3 where $83 \%$ of M2's were below $5 \mathrm{gl}^{-1}$ compared to the observed $24 \%$ in a prior MGUS study.

Serum levels of myeloma MABs are the same in MGMy and BGMy. The most prevalent M1 was IgG, followed by IgA, FLC and then IgD; no IgM or IgE M1's were observed; please refer to Supplementary Information for more information. The $\mathrm{HC}$ frequencies were very similar to those of 3248 patients in the same clinical trials who had MGMy (Table 1). Notably, IgG and IgA M1 MAB concentrations were not significantly different to those observed in the MGMy patients from the same trials. This indicates that the presence of a second neoplastic PC clone (i.e., M2) does not competitively suppress the expansion of the neoplastic myeloma clone (i.e., M1) (Table 3).

\section{DISCUSSION}

This is the largest study of BGMy frequency in a large cohort of newly diagnosed myeloma patients, and demonstrates that 
myeloma eliminates or greatly suppresses coexisting MGUS. As the prevalence of MGUS in the general population - at the typical age of myeloma diagnosis - is 5\% (Dispenzieri et al, 2010; Wadhera and Rajkumar, 2010) and the prevalence of BGUS amongst all individuals with MGUS is $>5 \%$ (Landgren et al, 2014), it would also be expected that $>5 \%$ of myeloma cases would be BGMy. However, despite rigorous central laboratory analysis, it was found to be just $0.91 \%$.

This shortfall is unlikely to be the result of myeloma being fivefold more likely to arise in individuals with a single MGUS clone than from individuals with two MGUS clones. A recent study reported the rate of progression from BGUS to myeloma was $\sim 1 \%$ per year, which is similar to the incidence of MGUS progression to myeloma (Mullikin et al, 2016). Thus, when myeloma arises from BGUS, in $\sim 80 \%$ of cases, the other MGUS clone must be eliminated or suppressed below the limits of detection on IFE $\left(0.1 \mathrm{gl}^{-1}\right)$.

In the BGMy cases reported herein, MGUS-associated MAB levels were smaller than expected levels typically observed in conventional MGUS (Turesson et al, 2014), by 2.5-fold for IgG and 5 -fold for IgA and IgM. The degree of suppression of MGUS clones in BGMy appears comparative to the immunoparesis of nonmalignant polyclonal PC that occurs in the majority of newly diagnosed myeloma patients (Pruzanski et al, 1980; Wangel, 1987; Wang and Young, 2001; Kastritis et al, 2014). Of 3248 MGMy patients from the same Myeloma IX and XI trials reported in this study, we found that polyclonal immunoglobulin levels were below the normal range in $>80 \%$ of patients and levels were lower than the median level found in healthy controls by 2.5 -fold for IgG and 7-fold for IgA and IgM. Despite their larger relative presence, MGUS clones appear to only compete effectively with polyclonal PC in $\sim 20 \%$ of cases, as evidenced by incidence of immunoparesis in MGUS (Turesson et al, 2014). As only a fifth of MGUS clones exhibit competitiveness for the normal PC niche, we hypothesise that these are the only MGUS clones that survive when myeloma arises in the wider marrow environment and that they are subject to the same suppression of clonal size and antibody secretion as normal polyclonal PCs; this would also be applicable to the MGUS PC clone from which myeloma arose. In a separate observation, we observed in this study that two IgG MABs was the most common BGMy combination (16 out of 58 patients) but not as common as expected (27 out of 58) indicating that the presence of IgG myeloma PC clones suppresses IgG MGUS PC clones more than IgA or IgM PC clones. There is evidence from both MGUS and myeloma that neoplastic IgG PC clones exert a greater degree of suppression on normal polyclonal IgG PC than they do on IgA and IgM isotypes (Bradwell et al, 2013; Katzmann et al, 2013; Ludwig et al, 2016).

A limitation of our study is that we were unable to conduct longitudinal measurement of BGUS before myeloma diagnosis; this excluded the possibility of investigating elimination of MGUS clones at the time of myeloma clone proliferation. Future prospective studies may seek to explore differences between BGUS that progress to myeloma, and BGUS that do not progress to myeloma, and measurements should incorporate bone marrow tumour samples as well as stroma to investigate possible mechanisms of tumour eradication. In summary, our findings confirm that BGMy is rare, and the survival of a coexisting MGUS clone is hallmarked by reduced MAB size.

\section{ACKNOWLEDGEMENTS}

We are grateful to the NCRI Haemato-oncology subgroup and to all principle investigators for their dedication and commitment to recruiting patients to Myeloma IX, XI and TEAMM trials. We thank the Clinical Trials Research Unit at The University of Leeds
(Myeloma IX and XI) and the Clinical Trials Unit at the University of Warwick (TEAMM). We are grateful to the staff of the Clinical Immunology Service in Birmingham with Tim Plant, Karen Walker, Alison Adkins and Nicola Newnham. Finally we are grateful to all patients and their clinical teams at centres throughout the UK whose participation made this study possible.

CONFLICT OF INTEREST

The authors declare no conflict of interest.

\section{AUTHOR CONTRIBUTIONS}

JPC, CMB and MTD wrote the manuscript; MTD, JPC and JLJH designed the investigation into BGMy; JPC, JLJH, SP and ZA conducted the experiments; JPC, JLJH, SP and MTD interpreted the data; MK, RO, GJM, GHJ, AC and MTD designed the Myeloma IX, $\mathrm{XI}$ and/or TEAMM trials. All authors approved the manuscript.

\section{REFERENCES}

Bradwell A, Harding S, Fourrier N, Mathiot C, Attal M, Moreau P, Harousseau JL, Avet-Loiseau H (2013) Prognostic utility of intact immunoglobulin Ig'kappa/Ig'lambda ratios in multiple myeloma patients. Leukemia 27(1): 202-207.

Dispenzieri A, Katzmann JA, Kyle RA, Larson DR, Melton 3rd LJ, Colby CL, Therneau TM, Clark R, Kumar SK, Bradwell A, Fonseca R, Jelinek DF, Rajkumar SV (2010) Prevalence and risk of progression of light-chain monoclonal gammopathy of undetermined significance: a retrospective population-based cohort study. Lancet 375(9727): 1721-1728.

Garcia-Garcia P, Enciso-Alvarez K, Diaz-Espada F, Vargas-Nunez JA, Moraru M, Yebra-Bango M (2015) Biclonal gammopathies: Retrospective study of 47 patients. Rev Clin Esp 215(1): 18-24.

Guastafierro S, Ferrara MG, Sica A, Parascandola RR, Santangelo S, Falcone U (2012) Serum double monoclonal components and hematological malignancies: only a casual association? Review of 34 cases. Leuk Res 36(10): 1274-1277.

Kastritis E, Zagouri F, Symeonidis A, Roussou M, Sioni A, Pouli A, Delimpasi S, Katodritou E, Michalis E, Michael M, Hatzimichael E, Vassou A, Repousis P, Christophoridou A, Kartasis Z, Stefanoudaki E, Megalakaki C, Giannouli S, Kyrtsonis MC, Konstantopoulos K, Spyroupoulou-Vlachou M, Terpos E, Dimopoulos MA, Greek Myeloma Study G (2014) Preserved levels of uninvolved immunoglobulins are independently associated with favorable outcome in patients with symptomatic multiple myeloma. Leukemia 28(10): 2075-2079.

Katzmann JA, Clark R, Kyle RA, Larson DR, Therneau TM, Melton 3rd LJ, Benson JT, Colby CL, Dispenzieri A, Landgren O, Kumar S, Bradwell AR, Cerhan JR, Rajkumar SV (2013) Suppression of uninvolved immunoglobulins defined by heavy/light chain pair suppression is a risk factor for progression of MGUS. Leukemia 27(1): 208-212.

Kyle RA, Gertz MA, Witzig TE, Lust JA, Lacy MQ, Dispenzieri A, Fonseca R, Rajkumar SV, Offord JR, Larson DR, Plevak ME, Therneau TM, Greipp PR (2003) Review of 1027 patients with newly diagnosed multiple myeloma. Mayo Clin Proc 78(1): 21-33.

Kyle RA, Robinson RA, Katzmann JA (1981) The clinical aspects of biclonal gammopathies. Review of 57 cases. Am J Med 71(6): 999-1008.

Kyle RA, Therneau TM, Rajkumar SV, Larson DR, Plevak MF, Offord JR, Dispenzieri A, Katzmann JA, Melton 3rd LJ (2006) Prevalence of monoclonal gammopathy of undetermined significance. $N$ Engl J Med 354(13): 1362-1369.

Landgren O, Graubard BI, Katzmann JA, Kyle RA, Ahmadizadeh I, Clark R, Kumar SK, Dispenzieri A, Greenberg AJ, Therneau TM, Melton 3rd LJ, Caporaso N, Korde N, Roschewski M, Costello R, McQuillan GM, Rajkumar SV (2014) Racial disparities in the prevalence of monoclonal gammopathies: a population-based study of 12,482 persons from the National Health and Nutritional Examination Survey. Leukemia 28(7): $1537-1542$. 
Landgren O, Kyle RA, Pfeiffer RM, Katzmann JA, Caporaso NE, Hayes RB, Dispenzieri A, Kumar S, Clark RJ, Baris D, Hoover R, Rajkumar SV (2009) Monoclonal gammopathy of undetermined significance (MGUS) consistently precedes multiple myeloma: a prospective study. Blood 113(22): 5412-5417.

Ludwig H, Milosavljevic D, Berlanga O, Zojer N, Hubl W, Fritz V, Harding S (2016) Suppression of the noninvolved pair of the myeloma isotype correlates with poor survival in newly diagnosed and relapsed/refractory patients with myeloma. Am J Hematol 91(3): 295-301.

Mullikin TC, Rajkumar SV, Dispenzieri A, Buadi FK, Lacy MQ, Lin Y, Dingli D, Go RS, Hayman SR, Zeldenrust SR, Russell SJ, Lust JA, Leung N, Kapoor P, Kyle RA, Gertz MA, Kumar SK (2016) Clinical characteristics and outcomes in biclonal gammopathies. Am J Hematol 91(5): 473-475.

Nilsson T, Norberg B, Rudolphi O, Jacobsson L (1986) Double gammopathies: incidence and clinical course of 20 patients. Scand J Haematol 36(1): 103-106.

Pruzanski W, Gidon MS, Roy A (1980) Suppression of polyclonal immunoglobulins in multiple myeloma: relationship to the staging and other manifestations at diagnosis. Clin Immunol Immunopathol 17(2): 280-286.

Riddell S, Traczyk Z, Paraskevas F, Israels LG (1986) The double gammopathies. Clinical and immunological studies. Medicine (Baltimore) 65(3): 135-142.
Turesson I, Kovalchik SA, Pfeiffer RM, Kristinsson SY, Goldin LR, Drayson MT, Landgren O (2014) Monoclonal gammopathy of undetermined significance and risk of lymphoid and myeloid malignancies: 728 cases followed up to 30 years in Sweden. Blood 123(3): 338-345.

Wadhera RK, Rajkumar SV (2010) Prevalence of monoclonal gammopathy of undetermined significance: a systematic review. Mayo Clin Proc 85(10): 933-942.

Wang L, Young DC (2001) Suppression of polyclonal immunoglobulin production by M-proteins shows isotype specificity. Ann Clin Lab Sci 31(3): 274-278.

Wangel A (1987) Multiple myeloma and polyclonal hypogammaglobulinaemia. Acta Med Scand 221(5): 421-425.

Weiss BM, Abadie J, Verma P, Howard RS, Kuehl WM (2009) A monoclonal gammopathy precedes multiple myeloma in most patients. Blood 113(22): 5418-5422.

This work is published under the standard license to publish agreement. After 12 months the work will become freely available and the license terms will switch to a Creative Commons AttributionNonCommercial-Share Alike 4.0 Unported License.

Supplementary Information accompanies this paper on British Journal of Cancer website (http://www.nature.com/bjc) 